\title{
Recurrence Relations and Hilbert Series of the Monoid Associated with Star Topology
}

\author{
Jiang-Hua Tang, ${ }^{1}$ Zaffar Iqbal, ${ }^{2}$ Abdul Rauf Nizami, ${ }^{3}$ Mobeen Munir $\mathbb{D}^{4},{ }^{4}$ Faiza Azam, \\ and Jia-Bao Liu $\left.{ }^{5}\right)^{5}$ \\ ${ }^{1}$ Department of General Education, Anhui Xinhua University, Hefei 230088, China \\ ${ }^{2}$ Department of Mathematics, University of Gujrat, Gujrat, Pakistan \\ ${ }^{3}$ University of Central Punjab, Department of Mathematics, Lahore, Pakistan \\ ${ }^{4}$ Department of Mathematics, Division of Science and Technology, University of Education Township, Lahore, Pakistan \\ ${ }^{5}$ School of Mathematics and Physics, Anhui Jianzhu University, Hefei 230601, China \\ Correspondence should be addressed to Mobeen Munir; mmunir@ue.edu.pk
}

Received 1 April 2020; Accepted 21 July 2020; Published 27 August 2020

Academic Editor: Ghulam Shabbir

Copyright (c) 2020 Jiang-Hua Tang et al. This is an open access article distributed under the Creative Commons Attribution License, which permits unrestricted use, distribution, and reproduction in any medium, provided the original work is properly cited.

\begin{abstract}
Affine monoids are the considered as natural discrete analogues of the finitely generated cones. The interconnection between these two objects has been an active area of research since last decade. Star network is one of the most common in computer network topologies. In this work, we study star topology $S_{n}$ and associate a Coxeter structure of affine type on it. We find a recurrence relation and the Hilbert series of the associated right-angled monoid $M\left(S_{n}^{\infty}\right)$. We observe that the growth rate of the monoid $M\left(S_{n}^{\infty}\right)$ is unbounded.
\end{abstract}

\section{Introduction}

Hilbert series of a graded commutative algebra is strongly related with dimensions of the homogeneous components of the algebra. This notion has been extended to filtered algebras and coherent sheaves over projective schemes [1]. Hilbert series is treated as a special case of Hilbert-Poincaré series of a graded vector space and is closely related with the number of words of an alphabet. Hilbert series helps counting words on an alphabet that do not contain a fixed set of words. This is also named as well-known forbidden subwords problem which can be translated at the level of monomial algebras. If we are given an alphabet $X=\left\{X_{1}, X_{2}, \ldots, X_{n}\right\}$ and words $W=\left\{W_{1}, W_{2}, \ldots, W_{d}\right\}$, then it is known that the numbers of words on $X$ avoiding this word $W$ is in one-to-one correspondence with monomials in $X$ having nonzero image in the ring $L=\mathbb{C}[X] / W$. If $L$ is a finitely generated monoid, then the coefficients of its Hilbert series satisfy a recursive relation. Hilbert series of several popular algebras appear as power series of rational functions [2]. Things become interesting especially when the monoid $L$ has a rational Hilbert series. This happens exactly when we have a finitely presented monoid. Significance of Hilbert series can be described in terms of the growth of a monoid. The growth of a monoid is said to be polynomially bounded if the $n$th coefficient of its Hilbert series is $0\left(n^{d}\right)$ for some number $d$. A monoid has exponential growth if the $n$th coefficient is larger than $C^{n}$ for some $C>1$. Interestingly, monoids should have polynomially bounded growths. The smallest-degree polynomial bounding the growth is one less than the Krull dimension. In noncommutative settings, topologists used growth to study fundamental groups. Milnor proved that if a compact Riemannian manifold has all its sectional curvatures negative, then its fundamental group has exponential growth, and if a complete $n$-dimensional Riemannian manifold has its mean curvature tensor everywhere positive semidefinite, then the finitely generated subgroup of its fundamental group has polynomially bounded growth. In short, a monoid has either an exponential growth or a polynomial growth. The degree of 
the growth is one less than the order of the pole at 1 of the Hibert series.

Coxeter groups were introduced by Canadian geometer H. S. M. Coxeter in 1934 to solve the well-known famous word problem, namely, whether two words occurring in generators of the presentation of groups correspond to same element or not. These groups have nice other properties such as having faithful linear representations as groups of reflections. In a nontrivial way, it can be proved that these groups are abstract analogues of the regular polytopes. These polytopes are convex-hull of some points in $\mathbb{R}^{n}$. Coxeter groups have generators $a_{i}, i \in I$ and have relations $a_{i}^{2}=1$ and $a_{i} a_{j} a_{i}=a_{j} a_{i} a_{j}$ with $i, j \in I$; finite and infinite groups are usually referred as spherical and affine.

Star topology is one of the important topologies used in networking and other real-world problems. One way to study this topology is by using Dynkin diagram [3] (or Coxeter graph), and other way is by using monoids. Note that the removal of the relations $a_{i}^{2}=1$ gives Artin groups. So, Coxeter groups are quotient groups of the Artin groups. A finite Coxeter group is a discrete acting group of reflections of a sphere [3]. That is why, they are known as spherical. The Artin braid group, $A_{n}$, is a spherical Coxeter groups. The Infinite Coxeter groups are generated by reflections in affine spaces [3].

In 2009, Saito [4] found spherical growth series of Artin monoids [5]. In [6], we gave a linear system for the canonical words of the braid monoid $M B_{n}$ which lead to find Hilbert series of $M B_{n}$. In [7], we computed Hilbert series of $M B_{4}$ in band generators. In 2006, Mairesse and Mathéus [8] gave dihedral-type growth series of Artin groups. In 1993, Parry [9] gave the growth series of Coxeter groups. In [10], we proved that the upper bound of the growth of spherical Artin monoids is 4 . But, in the affine case, this result is not true. In [11], we found a recurrence relation and Hilbert series of the associated right-angled affine Artin monoid $M\left(\widetilde{A}_{n}^{\infty}\right)$ and showed that its growth rate is unbounded. In [12], we found the Hilbert series of $M\left(\widetilde{D}_{n}^{\infty}\right)$ and showed that its growth rate is also unbounded.

In this paper, we study the star topology $S_{n}$ and find recurrence relations and the Hilbert series of the associated right-angled monoid $M\left(S_{n}^{\infty}\right)$. We also compute growth rate of the monoid $M\left(S_{n}^{\infty}\right)$ and observe that it is unbounded.

\section{Preliminaries}

We start this section with the notion of Coxeter groups and Artin groups. We study the star topology as a Dynkin diagram and then convert it as a monoid. These basic preliminary facts and notations which will be required later for formulating our main results.

Definition 1. A square symmetric matrix $M=\left(m_{s t}\right)_{s, t \in S}$ is said to be a Coxeter matrix over a nonempty set $S$ such that all the diagonal entries are 1 and $m_{s t} \in\{2,3,4, \ldots, \infty\}$.

Definition 2. Let $S$ be a set of vertices of a labeled graph $\Gamma$. We call $\Gamma$ a Coxeter graph if any two of its vertices are connected by an edge and if the label of each edge is greater than 2 .

By convention, each edge is labeled only if the label is greater than 3 .

Definition 3. A group with generator $s$ and relations $s^{2}=1$ and $(s t)^{m_{s t}}=1$ for all $s, t \in S$ and $m_{s t} \neq \infty$ is called a Coxeter group such that $\left(m_{s t}\right)_{s, t \in S}$ is the Coxeter matrix.

Definition 4. The Artin group is

$$
\mathscr{A}=\langle s \in S \mid \underbrace{s t s \ldots}_{m_{s t} \text { factors }}=\underbrace{t s t \cdots\rangle}_{m_{s t} \text { factors }}\rangle .
$$

If the Coxeter group is finite, then $\mathscr{A}$ is called a spherical Artin group.

Definition 5. The right-angled Artin groups or monoids are obtained if all the labels, which are greater than or equal to 3, of spherical Coxeter graphs are replaced with $\infty$.

The Artin spherical groups are usually represented by Coxeter graphs (see $[3,13]$ ); these groups are $A_{n}$ for $n \geq 1, B_{n}$ for $n \geq 2, D_{n}$ for $n \geq 4, E_{6}, E_{7}, E_{8}, F_{4}, G_{2}, H_{3}, H_{4}$, and $I_{2}(p)$ for $p \geq 5$ and $p \neq 6$. Figure 1 contains these graphs.

Definition 6 (see [14]). The length of a word $g=s_{1}, \cdots, s_{n}$ of a finitely generated group $G$ is the smallest nonnegative integer $n$ for which $s_{1}, \ldots, s_{n} \in S \cup S^{-1}$, where $S$ is the set of generators of $G$.

Definition 7 (see [14]). The spherical growth series of a finitely generated group $G$ is $H_{G}(t)=\sum_{k=0}^{\infty} a_{k} t^{k}$, where $a_{k}$ is the number of words of length $k$.

Let $a=b$ be a relation in a given monoid $M$. Then, in length-lexicographic order, $a$ is greater then or equal to $b$. A word $u w v$ has an ambiguity if $u w$ and $w v$ are left sides two relations. If $\alpha_{1} v$ and $u \alpha_{2}$ are identical, then $u w v$ is solvable. If $\alpha_{1} v$ and $u \alpha_{2}$ differ by lexicographic order, then we get a new relation in $M$. A presentation is said to be complete if solutions of all ambiguities are identical. A reducible word is the left side of a relation of a complete presentation of a monoid. If $w$ does not contain the LHS of any relation, then $w$ is called a canonical word. The following notions are in [15-20] under different terminologies: Gröbner bases, complete presentation, rewriting system, and so on.

\section{Main Results}

In this part, we compute our main results.

3.1. Recurrence Relation of the Monoid $M\left(S_{n}^{\infty}\right)$. In this paper, we study the star topology $S_{n}$ and find recurrence relations and the Hilbert series of the associated right-angled monoid $M\left(S_{n}^{\infty}\right)$. We compute the growth rate of the monoid $M\left(S_{n}^{\infty}\right)$, and using the graph, we show that it is unbounded. The Coxeter graph of the star topology $S_{n}$ is given by the following graph (Figure 2): 


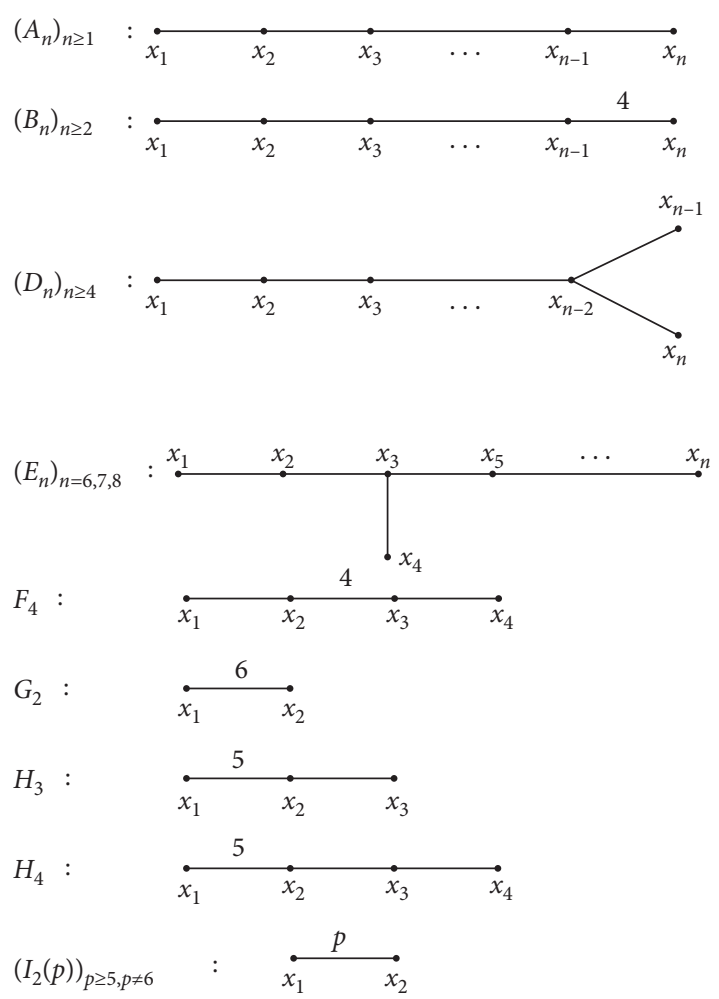

Figure 1: Spherical Coxeter graphs.

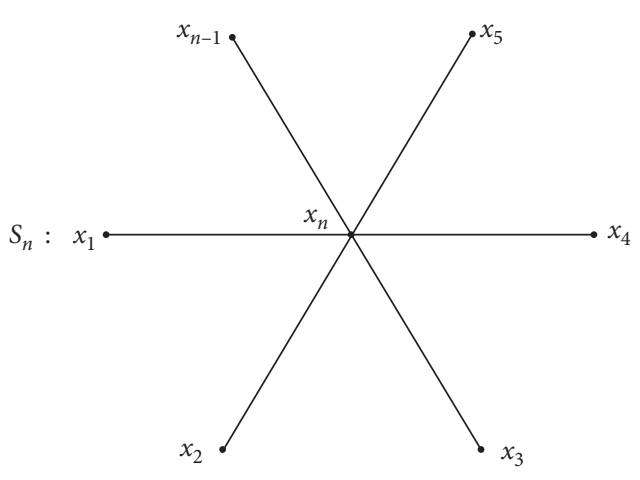

Figure 2: $S_{n}$.

We denote the right-angled monoid associated with $S_{n}$ by $M\left(S_{n}^{\infty}\right)$. In $M$, we fix a total order $x_{1}<x_{2}<\cdots<x_{n}$ on the generators. Hence, clearly we have the following lemma.

Lemma 1. The monoid $M\left(S_{n}^{\infty}\right)$ has generators $x_{1}, x_{2}, \ldots, x_{n}$ and relations $x_{i} x_{j}=x_{j} x_{i}$ for $1 \leq j+1 \leq i \leq n-1$ and $x_{n} x_{k}=$ $x_{k} x_{n}$ for $2 \leq k \leq n-2$.

This section covers some useful results about recursive relations of $M\left(S_{n}^{\infty}\right)$ : Consider a system [21] of linear relations:

$$
\begin{aligned}
u_{i}(t+1)= & a_{i 1}(t) u_{1}(t)+a_{i 2}(t) u_{2}(t)+\cdots+a_{i n}(t) u_{n}(t) \\
& +f_{i}(t), \quad 1 \leq i \leq n .
\end{aligned}
$$

Equivalently, $u(t+1)=A(t) u(t)+f(t)$, where

$$
\begin{aligned}
u(t) & =\left[\begin{array}{c}
u_{1}(t) \\
\vdots \\
u_{n}(t)
\end{array}\right], \\
A(t) & =\left[\begin{array}{ccc}
a_{11}(t) & \cdots & a_{1 n}(t) \\
\vdots & \ddots & \vdots \\
a_{n 1}(t) & \cdots & a_{n n}(t)
\end{array}\right], \\
f(t) & =\left[\begin{array}{c}
f_{1}(t) \\
\vdots \\
f_{n}(t)
\end{array}\right] .
\end{aligned}
$$

The solution of the system $u(t+1)=A(t) u(t)$ is $u(t)=c_{1} \lambda_{1}^{t} u^{1}+\cdots+c_{k} \lambda_{k}^{t} u^{k}$, where $\lambda_{i}$ and $u^{i}, 1 \leq i \leq k$ are, respectively, the eigenvalues and eigenvectors of $A(t)$. The largest eigenvalue represents the growth rate of the sequence $u_{1}(t), u_{2}(t), \ldots, u_{k}(t)$.

In the following, by $c_{k}$ and $c_{k ; i}$, we shall mean the number of canonical words of length $k$ and words starting with $x_{i}$.

Lemma 2. $M\left(S_{n}^{\infty}\right)$ satisfies the relations $c_{0}=1, c_{1 ; i}=1$, and $c_{k}=\sum_{i=1}^{n} c_{k ; i} \quad(k \geq 1)$, where $c_{k ; i}$ is

$$
c_{k ; i}= \begin{cases}\sum_{j=i}^{n} c_{k-1 ; j}, & 1 \leq i \leq n-1, \\ c_{k ; 1}, & i=n .\end{cases}
$$

Let $S_{n}(\lambda)$ denote the characteristic polynomial, then we have the following.

Theorem 1. The characteristic polynomial $S_{n}(\lambda)$ of the system of recursive relations of $M\left(S_{n}^{\infty}\right)$ satisfies the relation:

$$
S_{n}(\lambda)=\lambda S_{n-1}(\lambda)-\lambda(\lambda-1)^{n-2},
$$

where $n \geq 2$ and $S_{1}(\lambda)=\lambda-1$.

Proof. The characteristic polynomial of the coefficient matrix of the system of recurrence relations given in Lemma 2 is

$$
S_{n}(\lambda)=\left|\begin{array}{ccccccc}
\lambda-1 & -1 & \cdots & -1 & -1 & -1 & -1 \\
0 & \lambda-1 & \cdots & -1 & -1 & -1 & -1 \\
\vdots & \vdots & & \vdots & \vdots & \vdots & \vdots \\
0 & 0 & \cdots & 0 & \lambda-1 & -1 & -1 \\
0 & 0 & \cdots & 0 & 0 & \lambda-1 & -1 \\
-1 & -1 & \cdots & -1 & -1 & -1 & \lambda-1
\end{array}\right| .
$$




$$
S_{n}(\lambda)=\left|\begin{array}{ccccccc}
\lambda-1 & -1 & \cdots & -1 & -1 & -1 & -1 \\
0 & \lambda-1 & \cdots & -1 & -1 & -1 & -1 \\
\vdots & \vdots & & \vdots & \vdots & \vdots & \vdots \\
0 & 0 & \cdots & 0 & \lambda-1 & -1 & -1 \\
-1 & -1 & \cdots & -1 & -1 & \lambda-2 & \lambda-2 \\
-1 & -1 & \cdots & -1 & -1 & -1 & \lambda-1
\end{array}\right|
$$

Now, subtracting the 2nd last column from the last column and simplifying, we have $S_{n}(\lambda)=\lambda T_{n-1}(\lambda)$, where

$$
T_{n-1}(\lambda)=\left|\begin{array}{cccccc}
\lambda-1 & -1 & \cdots & -1 & -1 & -1 \\
0 & \lambda-1 & \cdots & -1 & -1 & -1 \\
\vdots & \vdots & & \vdots & \vdots & \vdots \\
0 & 0 & \cdots & -1 & \lambda-1 & -1 \\
-1 & -1 & \cdots & -1 & -1 & \lambda-2
\end{array}\right| .
$$

We write $T_{n-1}(\lambda)=U_{n-1}(\lambda)+V_{n-1}(\lambda)$, where the determinants $U_{n-1}(\lambda)$ and $V_{n-1}(\lambda)$ are obtained by splitting $T_{n-1}(\lambda)$ such that the last row of $U_{n-1}(\lambda)$ is $(-1, \ldots,-1, \lambda-$ $1)$ and the last row of $V_{n-1}(\lambda)$ is $(0, \ldots, 0,-1)$. Hence, easily, we have $U_{n-1}(\lambda)=S_{n-1}(\lambda)$ and $V_{n-1}(\lambda)=-(\lambda-1)^{n-2}$. Therefore, we have

$$
S_{n}(\lambda)=\lambda S_{n-1}(\lambda)-\lambda(\lambda-1)^{n-2} .
$$

Here, we have an explicit formula for $S_{n}(\lambda)$.

Lemma 3. In $M\left(S_{n}^{\infty}\right)$, characteristic polynomial is given explicitly by

$$
S_{n}(\lambda)=\lambda^{n-1}(\lambda-1)-\sum_{i=1}^{n-1} \lambda^{i}(\lambda-1)^{n-i-1} .
$$

Proof. From equation (5), we have $S_{n-k}(\lambda)=\lambda S_{n-k-1}(\lambda)-\lambda(\lambda-1)^{n-k-2}, k \geq 0$. Hence, we have

$$
\begin{aligned}
S_{n}(\lambda) & =\lambda S_{n-1}(\lambda)-\lambda(\lambda-1)^{n-2} \\
& =\lambda^{2} S_{n-2}(\lambda)-\lambda(\lambda-1)^{n-2}-\lambda^{2}(\lambda-1)^{n-3} \\
& =\lambda^{3} S_{n-3}(\lambda)-\lambda(\lambda-1)^{n-2}-\lambda^{2}(\lambda-1)^{n-3}-\lambda^{3}(\lambda-1)^{n-4} \\
& =\lambda^{n-1} S_{n-(n-1)}-\sum_{i=1}^{n-1} \lambda^{i}(\lambda-1)^{n-i-1} \\
& =\lambda^{n-1}(\lambda-1)-\sum_{i=1}^{n-1} \lambda^{i}(\lambda-1)^{n-i-1} .
\end{aligned}
$$

3.2. Hilbert Series of the Monoid $M\left(S_{n}^{\infty}\right)$. From now on, $H_{M}^{(n)}(t)=\sum_{k \geq 0} c_{k} t^{k}$ will denote the Hilbert series of $M\left(S_{n}^{\infty}\right)$, and $H_{M ; i}^{(n)}(t)=\sum_{k \geq 1} c_{k: i}^{(n)} t^{k}$ will denote the Hilbert series of $M\left(S_{n}^{\infty}\right)$ of the words starting with $x_{i}$.
Following Lemma 2 we get the following.

Theorem 2. For $M\left(S_{n}^{\infty}\right)$, we have

(1) $H_{M}^{(n)}(t)=1+\sum_{i=1}^{n} H_{M: i}^{(n)}(t)$

(2) $H_{M ; i}^{(n)}(t)=t+t \sum_{j=i}^{n} H_{M ; j}^{(n)}(t), 1 \leq i \leq n-1$

(3) $H_{M ; 1}^{(n)}(t)=H_{M ; n}^{(n)}(t)$

Proof

(1) Since $c_{k}=\sum_{i=1}^{n} c_{k ; i},(k \geq 1), H_{M}^{n}(t)=\sum_{k \geq 0} c_{k} t^{k}=c_{0}+$ $\sum_{k \geq 1} c_{k} t^{k}=1+\sum_{k \geq 1} \sum_{i=1}^{n} c_{k ; i} t^{k}=1+\sum_{i=1}^{n} \sum_{k \geq 1} c_{k ; i} t^{k}=$ $1+\sum_{i=1}^{n} H_{M ; i}^{n}(t)$.

(2) Also, from Lemma 2, we have $c_{k ; i}=\sum_{j=i}^{n} c_{k-1 ; j}$ $(1 \leq i \leq n-1)$. Hence, $H_{M ; i}^{(n)}(t)=\sum_{k \geq 1}^{n} c_{k ; i}(t) t^{k}=$ $c_{1 ; i}(t) t+\sum_{k \geq 2}^{n} c_{k ; i}(t) t^{k}=t+\sum_{k \geq 2}^{n} \sum_{j=i}^{n} c_{k-1 ; j}(t) t^{k}=$ $t+t \sum_{j=i}^{n} \sum_{k \geq 2}^{n} c_{k-1 ; j}(t) t^{k-1}=t+t \sum_{j=i}^{n} H_{M ; j}^{(n)}(t)$.

(3) It can be proved similarly.

The linear system of Theorem 2 takes the form $W_{n} X=B$, where

$$
\begin{aligned}
& W_{n}=\left[\begin{array}{cccccc}
1-t & -t & \cdots & -t & -t & -t \\
0 & 1-t & \cdots & -t & -t & -t \\
0 & 0 & \cdots & -t & -t & -t \\
\vdots & \vdots & & \vdots & \vdots & \vdots \\
0 & 0 & \cdots & 0 & 1-t & -t \\
-t & -t & \cdots & -t & -t & 1-t
\end{array}\right], \\
& X=\left[\begin{array}{c}
H_{M ; 1}^{(n)}(t) \\
H_{M ; 2}^{(n)}(t) \\
\vdots \\
H_{M ; n}^{(n)}(t)
\end{array}\right] \text {, } \\
& B=\left[\begin{array}{c}
t \\
t \\
\vdots \\
t
\end{array}\right] .
\end{aligned}
$$

Lemma 4. For $M\left(S_{n}^{\infty}\right)$, we have $H_{M ; m}^{(n)}(t)=((t)(1-$ $\left.\left.t)^{m-1}\right) / t^{n} S_{n}(1 / t)\right), m=1,2, \ldots, n-1$.

For the system of equations given in Theorem 2, we have the following.

\section{Lemma 5}

$$
\operatorname{det}\left(W_{n}\right)=t^{n} S_{n}\left(\frac{1}{t}\right)
$$

Proof. It is obvious to Just factor out $t$ from each row of $\operatorname{det}\left(W_{n}\right)$. 


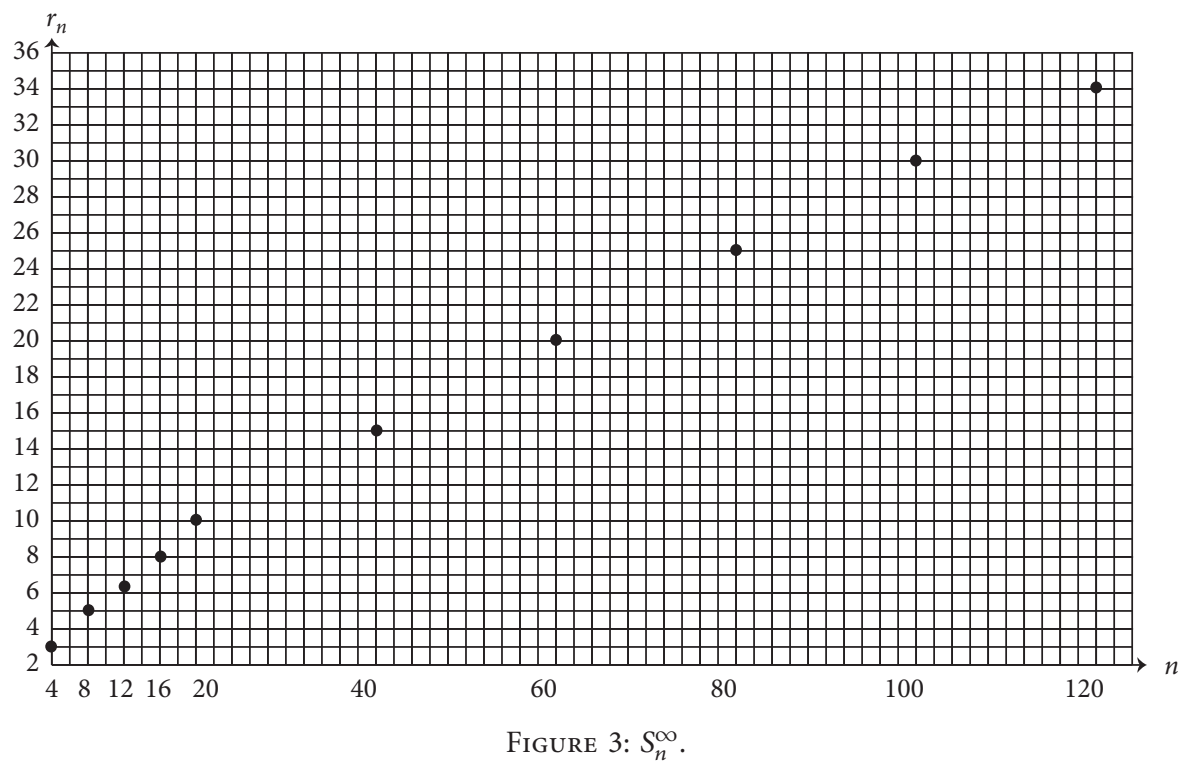

\section{Lemma 6}

$$
H_{M ; m}^{(n)}(t)=\frac{t(1-t)^{m-1}}{t^{n} S_{n}(1 / t)}, \quad m=1,2, \ldots, n-1 .
$$

Proof. The solution of the system $W_{n} X=B$ is $H_{M ; m}^{(n)}(t)=\left(T_{m} / \operatorname{det}\left(W_{n}\right)\right)$, where $T_{m}$ is the determinant of the matrix obtained by replacing 0 in the $m$ th column of $W_{n}$ by $B$. That is,

$$
T_{m}=\left|\begin{array}{cccccccc}
1-t & -t & \cdots & -t & t & \cdots & -t & -t \\
0 & 1-t & \cdots & -t & t & \cdots & -t & -t \\
0 & 0 & \cdots & -t & t & \cdots & -t & -t \\
\vdots & \vdots & & \vdots & \vdots & & \vdots & \vdots \\
0 & 0 & \cdots & 0 & t & \cdots & 0 & 1-t
\end{array}\right| .
$$

Adding $m$ th column of $T_{m}$ to its column numbers $m+1, m+2, \ldots, n, \quad$ we $\quad$ get $H_{M ; m}^{(n)}(t)=$ $\left(\left(t(1-t)^{m-1}\right) / t^{n} S_{n}(1 / t)\right), m=1,2, \ldots, n-1$.

The following result gives the Hilbert series of $M\left(S_{n}^{\infty}\right)$.

\section{Theorem 3}

$$
H_{M}^{(n)}(t)=\frac{1}{t^{n} S_{n}(1 / t)} .
$$

Proof. Equation (5) gives $t^{n} S_{n}(1 / t)=t^{n-1} S_{n-1}(1 / t)$ $-t(1-t)^{n-2}$. Hence, Lemma 6 and Theorem 2 imply that

$$
\begin{aligned}
H_{M}^{(n)}(t)= & 1+H_{M ; 1}^{(n)}(t)+H_{M ; 2}^{(n)}(t)+\cdots+H_{M ; n-1}^{[n)}(t) \\
& +H_{M ; n}^{(n)}(t) \\
= & \frac{1}{t^{n} S_{n}(1 / t)}\left(t^{n} S_{n}\left(\frac{1}{t}\right)+t+t(1-t)\right. \\
& \left.+t(1-t)^{2}+\cdots+t(1-t)^{n-2}+t\right) \\
= & \frac{1}{t^{n} S_{n}(1 / t)}\left(t^{n-1} S_{n-1}\left(\frac{1}{t}\right)+2 t+t(1-t)\right. \\
& \left.+t(1-t)^{2}+\cdots+t(1-t)^{n-3}\right) \\
= & \frac{t S_{1}(1 / t)-t}{t^{n} S_{n}(1 / t)} \\
= & \frac{1}{t^{n} S_{n}(1 / t)} .
\end{aligned}
$$

3.3. Conclusions. Here, we gave, by constructing the affinetype Coxeter structure on star topology, a recursive relation and the Hilbert series of the right-angled monoid $M\left(S_{n}^{\infty}\right)$ associated with a star graph. The main result is $H_{M}^{(n)}(t)=\left(1 / t^{n} S_{n}(1 / t)\right)$. We also computed the growth rate, $r_{n}$, of $M\left(S_{n}^{\infty}\right)$ using Mathematica; some initial values are: $r_{3}=2.618, r_{4}=3.147, r_{5}=3.629, r_{6}=4.079, r_{7}=4.506$, $r_{8}=4.915, r_{9}=5.309, r_{10}=5.691, r_{11}=6.063, r_{12}=6.426$, 
$r_{13}=6.781, \quad r_{14}=7.130, \quad r_{15}=7.472, \quad r_{16}=7.809$, $r_{17}=8.141, r_{18}=8.468, r_{19}=8.790, r_{20}=9.109$. We also compute $r_{40}=14.93, r_{60}=20.15, r_{80}=25.04, r_{100}=29.7$, and $r_{120}=34.2$. We have the following graph representing the behavior of the growth rate of $S_{n}(\lambda)$ (Figure 3)

We observe that the growth rate for $M\left(S_{n}^{\infty}\right)$ increases and is unbounded. Hence, at the end, we have the following natural open problem emerging from our research.

An open problem: the growth rate of $M\left(S_{n}^{\infty}\right)$ is unbounded.

\section{Data Availability}

No such data are used in this research.

\section{Conflicts of Interest}

The authors declare that they have no conflicts of interest.

\section{Acknowledgments}

This project was supported by the Natural Science Fund Project of Anhui Xinhua University ( Grant No. 2017zr011).

\section{References}

[1] R. P. Stanley, "Hilbert functions of graded algebras," Advances in Mathematics, vol. 28, no. 1, pp. 57-83, 1978.

[2] S. Hal, Computational Algebraic Geometry, Cambridge University Press, Cambridge, UK, 2003.

[3] N. Bourbaki, Groupes et algèbres de Lie, Chapitres 4-6, Elementary Mathematics, Hermann, MO, USA, 1968.

[4] K. Saito, "Growth functions for Artin monoids," Proceedings of the Japan Academy, Series A, Mathematical Sciences, vol. 85, no. 7, pp. 84-88, 2009.

[5] E. Artin, "Theory of braids," The Annals of Mathematics, vol. 48, no. 1, pp. 101-126, 1947.

[6] Z. Iqbal, "Hilbert series of positive braids," Algebra Colloquium, vol. 18, pp. 1017-1028, 2011.

[7] Z. Iqbal and S. Yousaf, "Hilbert series of the braid monoid \$MB_\{4\}\$ in band generators," Turkish Journal of Mathematics, vol. 38, pp. 977-984, 2014.

[8] J. Mairesse and F. Mathéus, "Growth series for Artin groups of dihedral type," International Journal of Algebra and Computation, vol. 16, no. 6, pp. 1087-1107, 2006.

[9] W. Parry, "Growth series of Coxeter groups and salem numbers," Journal of Algebra, vol. 154, no. 2, pp. 406-415, 1993.

[10] B. Berceanu and Z. Iqbal, "Universal upper bound for the growth of Artin monoids," Communications in Algebra, vol. 43, no. 5, pp. 1967-1982, 2015.

[11] Z. Iqbal, S. Batool, and M. Akram, "Hilbert series of rightangled affine Artin monoid $M\left(\widetilde{A}_{n}^{\infty}\right)$," Kuwait Journal of Science, vol. 44, no. 4, pp. 19-27, 2017.

[12] C. Young, Z. Iqbal, A. Rauf Nizami, M. Munir, S. Riaz, and M. Shin, "Some recurrence relations and Hilbert series of right-angled affine Artin monoid $M\left(\widetilde{D}_{n}^{\infty}\right)$," Journal of Function Spaces, vol. 2018, Article ID 1901657, 6 pages, 2018.

[13] H. S. M. Coxeter, Regular Complex Polytopes, Cambridge University Press, Cambridge, UK, 2nd edition, 1991.

[14] P. D. Harpe, Topics in Geometric Group Theory, The University of Chicago Press, Chicago, IL, USA, 2000.
[15] D. J. Anick, "On the homology of associative algebras," Transactions of the American Mathematical Society, vol. 296, no. 2, p. 641, 1986.

[16] G. M. Bergman, "The diamond lemma for ring theory," Advances in Mathematics, vol. 29, no. 2, pp. 178-218, 1978.

[17] L. A. Bokut, Y. Fong, W.-F. Ke, and L.-S. Shiao, "GröbnerShirshov bases for braid semigroup," in Advances in Algebra, pp. 60-72, World Scientific Publishing, Singapore, 2003.

[18] K. S. Brown, "The geometry of rewriting systems:a proof of Anick-Groves-Squeir theorem," in Algorithms and Classification in Combinatorial Group Theory, G. Baumslag and C. F. Miller, Eds., pp. 137-164, Springer, New York, NY, USA, 1992.

[19] P. M. Cohn, Further Algebra and Applications, Springer, London, UK, 2003.

[20] V. A. Ufnarovskij, "Combinatorial and asymptotic methods in algebra," in Encyclopaedia of Mathematical Sciences, Springer, Berlin, Germany, 1995.

[21] W. G. Kelley and A. C. Peterson, Difference equations: An Introduction with Applications, p. 125, Second edition, Academic Press, New York, NY, USA, 2001. 